\title{
PKM2 regulates proliferation and apoptosis through the Hippo pathway in oral tongue squamous cell carcinoma
}

\author{
JIA LUO $^{1 *}$, LEI ZHANG ${ }^{2 *}$, LIJUAN GUO $^{3}$ and SEN YANG ${ }^{1}$ \\ ${ }^{1}$ Department of Oral and Maxillofacial Surgery, Suining Central Hospital, Suining, Sichuan 629000; \\ ${ }^{2}$ Department of Endodontics, The First Affiliated Hospital of Harbin Medical University, Harbin, Heilongjiang 150001; \\ ${ }^{3}$ Medical Beauty Department, Suining Central Hospital, Suining, Sichuan 629000, P.R. China
}

Received November 27, 2020; Accepted March 16, 2021

DOI: $10.3892 / \mathrm{ol} .2021 .12722$

\begin{abstract}
Oral tongue squamous cell carcinoma (OTSCC) is a highly malignant type of tumor. The 5-year survival rate of patients with advanced tongue squamous cell carcinoma is only $\sim 50 \%$. Pyruvate kinase M2 (PKM2) is the key rate-limiting enzyme of glycolysis, maintaining the Warburg effect in tumor cells. The present study aimed to investigate the relationship between PKM2 expression and the poor prognosis of patients with OTSCC and to determine oral squamous carcinoma tumor cell proliferation and apoptosis. Reverse transcription-quantitative (RT-q) PCR, western blotting and immunohistochemistry were used to analyze the expression levels of PKM2 in OTSCC, and the clinicopathological characteristics and prognosis of patients with OTSCC were further analyzed by statistical analysis. The results from RT-qPCR and immunohistochemistry demonstrated that PKM2 was upregulated in OTSCC tissues and highly expressed in advanced stage OTSCC tissues compared with paired adjacent tissues and lower stage OTSCC tissues. Patients with OTSCC and high PKM2 expression had shorter overall survival (OS) compared with those with low PKM2 expression. Furthermore, high expression of PKM2 was significantly associated with Tumor-Node-Metastasis (TNM) stage. TNM stage and PKM2 expression were independent predictive factors for OS in patients with OTSCC. In addition, PKM2 knockdown inhibited the proliferation and increased the apoptosis of oral squamous carcinoma tumor cells.
\end{abstract}

Correspondence to: Professor Sen Yang, Department of Oral and Maxillofacial Surgery, Suining Central Hospital, 127 West Desheng Road, Suining, Sichuan 629000, P.R. China

E-mail: senyang5@126.com

Professor Lijuan Guo, Medical Beauty Department, Suining Central Hospital, 127 West Desheng Road, Chuanshan, Suining, Sichuan 629000, P.R. China

E-mail: drguolijuan@yahoo.com

*Contributed equally

Key words: oral tongue squamous cell carcinoma, pyruvate kinase M2, Hippo pathway, proliferation, apoptosis
Furthermore, PKM2 knockdown could regulate the expression of cell cycle and apoptosis-related proteins by activating Hippo signaling pathway, as confirmed by the decreased expression of yes-associated protein 1 (YAP), Bcl-2 and Ki-67 and the increased expression of large tumor suppressor kinase 1, phosphorylated YAP and Bax. Taken together, the findings from this study demonstrated that PKM2 may be considered as a potential target for the diagnosis and treatment of OTSCC.

\section{Introduction}

Oral tongue squamous cell carcinoma (OTSCC) is difficult to detect because of its subtle early symptoms. The incidence of OTSCC is caused by long-term mechanical and chemical irritation of the oral cavity (1). The early symptoms of OTSCC include foreign body sensation or swallowing pain. With tumor growth, dysphagia, unclear enunciation and deep ear pain may occur, resulting in heavy physical and mental burdens $(2,3)$. In recent years, with the application of systemic chemotherapy and radiotherapy, the prognosis of patients has significantly improved; however, the 5-year survival rate remains low (4). It is therefore crucial to identify novel key molecules that could provide scientific targets for accurate diagnosis and precise treatment of OTSCC, to ultimately improve the poor prognosis of patients.

Pyruvate kinase (PK) is the ultimate rate-limiting enzyme of glycolysis, and the M2 splicing subtype of pyruvate kinase (PKM2) is the key enzyme involved in the Warburg effect, which participates in the transfer of phosphoenolpyruvate to adenosine diphosphate (ATP) $(5,6)$. Accumulating evidence demonstrated that PKM2 is highly expressed in many malignant tumors, such as liver, gastric and colon cancers, and that its high expression is a necessary condition to ensure Warburg effect $(7,8)$. The Warburg effect allows tumor cells to replenish their production capacity in the microenvironment, which is characterized by hypoxia and mitochondrial oxidative phosphorylation. The resulting high ATP/ADP ratio induces a rapid proliferation of tumor cells and promotes malignant signal transduction mechanisms (9). Phosphorylation of PKM2 at tyrosine 105 (Y105) activates Yes-associated protein (YAP), which then increases cancer stem-like cell properties through Warburg effect to promote the malignant proliferation of breast cancer cells (10). In addition, by interacting with inositol-1,4,5-triphosphate receptor, methylated PKM2 can 
inhibit the influx of calcium from endoplasmic reticulum to mitochondrion, which leads to an increase in tumor cell proliferation, migration and metastasis (11). However, the effects of PKM2 on OTSCC have not been established.

The present study aimed to investigate the expression of PKM2 in OTSCC tissues, and to determine its prognostic value in patients with OTSCC. In addition, we analyzed the effect of PKM2 on the proliferation and apoptosis of OTSCC cells.

\section{Materials and methods}

Patients and samples. A total of 125 OTSCC tumor tissues and paired adjacent tissues ( $>5 \mathrm{~cm}$ from the boundary of the tumor) were collected from patients who underwent surgical resection between June 2011 and August 2013 at the Suining Central Hospital. The clinicopathological characteristics of patients were obtained from the Suining Central Hospital. All clinical samples were stored in liquid nitrogen (12). A follow-up was implemented to evaluate the survival rate of patients 5 years post-surgery. This study was approved by the Ethics Committee of the Suining Central Hospital (approval no. 20110067, Suining, China) and was conducted in accordance with the Declaration of Helsinki.

Cell lines and transfection. The human oral squamous carcinoma cell lines SCC-9, SCC-15, the human tongue squamous cell carcinoma cell line $\mathrm{H} 357$ and the normal embryonic bovine tracheal epithelial cell line EBTr were obtained from the American Type Culture Collection. All cells were cultured in Dulbecco's modified Eagle's medium (Thermo Fisher Scientific, Inc.) supplemented with 10\% FBS (Gibco; Thermo Fisher Scientific, Inc.) and 100 units/ml penicillin and streptomycin (Gibco; Thermo Fisher Scientific, Inc.) and placed at $37^{\circ} \mathrm{C}$ in a humidified incubator containing $5 \% \mathrm{CO}_{2}$.

A total of $1 \times 10^{5}$ SCC-9 and H357 cells cultured in serum free DMEM were transfected with $100 \mathrm{nM}$ of small interfering (si)-PKM2 and si-negative control (NC; Guangzhou RiboBio Co., Ltd.) according to the manufacturers' instructions using Lipofectamine ${ }^{\circledR} 3000$ reagent (Invitrogen; Thermo Fisher Scientific, Inc.) at $37^{\circ} \mathrm{C}$. The transfection efficiency was confirmed using RT-qPCR $24 \mathrm{~h}$ after transfection and transfected cells were used for following experiments.

$R N A$ extraction and reverse transcription quantitative $(R T-q)$ $P C R$. Total RNA from OTSCC tissues, adjacent tissues and cell lines was extracted using TRIzol ${ }^{\mathrm{TM}}$ reagent (Takara Bio, Inc.). Total RNA was reverse transcribed into cDNA using a PrimeScript RT Reagent kit (Takara Bio, Inc.) at $37^{\circ} \mathrm{C}$ for $10 \mathrm{~min}, 45^{\circ} \mathrm{C}$ for $30 \mathrm{~min}$ and $75^{\circ} \mathrm{C}$ for $10 \mathrm{~min}$. PrimeScript RT Reagent (Takara Bio, Inc.) was used for reverse transcription and RT-qPCR was performed using SYBR Premix Ex Taq II (Takara Bio,Inc.) and a LightCycler system (Roche Diagnostics). The sequences of the primers were as follows: PKM2, forward 5'-GGGTTCGGAGGTTTGATG-3' and reverse 5'-ACGGCG GTGGCTTCTGT-3'; and GAPDH, forward 5'-ATGTTGCAA CGGGAAGGA-3' reverse 5'-AGGAAAAGCATCACCCGG AG-3'. The following thermocycling conditions were used for the qPCR: Initial denaturation at $90^{\circ} \mathrm{C}$ for $2 \mathrm{~min}$; followed by 40 cycles at $95^{\circ} \mathrm{C}$ for $15 \mathrm{sec}, 63^{\circ} \mathrm{C}$ for $30 \mathrm{sec}$ and $75^{\circ} \mathrm{C}$ for $30 \mathrm{sec}$. The relative expression levels were normalized to endogenous control and were expressed as $2^{-\Delta \Delta \mathrm{Cq}}(13)$.
Immunohistochemistry (IHC) and immunohistochemical staining. Tissue microarrays (TMA) were prepared from the 125 pairs of OTSCC and adjacent tissues. Briefly, tissues were fixed in $10 \%$ formalin for $12 \mathrm{~h}$ at room temperature and embedded in paraffin blocks for $8 \mathrm{~h}$ at room temperature. TMA cores (1.5-mm diameter) were constructed from formalin-fixed paraffin-embedded OTSCC and adjacent tissues sections (4- $\mu \mathrm{m}$ thick). Tissue sections were rehydrated in xylene and alcohol $(100,95$ and $80 \%)$ and incubated with $3 \% \mathrm{H}_{2} \mathrm{O}_{2}$ for $30 \mathrm{~min}$ at $37^{\circ} \mathrm{C}$. All sections were incubated for $15 \mathrm{~min}$ with $5 \%$ goat serum (OriGene Technologies, Inc.) to block nonspecific binding, followed by incubation with a rabbit monoclonal PKM2 antibody (cat. no. ab38237; 1:100; Abcam) at $4^{\circ} \mathrm{C}$ overnight. Sections were subsequently incubated with anti-rabbit secondary $\operatorname{IgG}$ antibody (1:100; cat. no. SAP-9100; OriGene Technologies, Inc.) at $37^{\circ} \mathrm{C}$ for $30 \mathrm{~min}$. After washing with PBS, visualization was achieved using diaminobenzidine (Boster Biological Technology).

PKM2 immunostaining was scored and examined by two independent assessors. To determine the final staining scores, all tissues were manually scored using the percentage of positively stained cells and the intensity of the staining. The scoring parameters included the staining intensity and the percentage of positive cells. The staining intensity ranged from 0-3 as follows: 0 , negative; 1 , weak; 2 , moderate; and 3 , strong. The percentage of positive cells ranged from $0-4$ as follows: 0 , negative or $<5 ; 1,6-25 ; 2,26-50 ; 3,51-75$; and $4,76-100 \%$. The total score was equal to the sum of staining intensity and percentage of positive cells. Slides with a total score $<4$ were defined as low PKM2 expression, while slides with a score $\geq 4$ were defined as high PKM2 expression (14).

Western blotting. Total protein was extracted using RIPA lysis buffer (Beyotime Institute of Biotechnology). Total protein was quantified using the Bradford protein assay (Bio-Rad Laboratories, Inc.) and $40 \mu \mathrm{g}$ protein/lane was separated via $10 \%$ SDS-PAGE. The separated proteins were subsequently transferred onto PVDF membranes that were blocked with $5 \%$ skim milk powder at room temperature for $1 \mathrm{~h}$. The membranes were incubated with the following primary antibodies at $4^{\circ} \mathrm{C}$ overnight: Anti-PKM2 (1:2,000; cat. no. ab85555; Abcam), anti-large tumor suppressor kinase 1 (LATS1; 1:5,000; cat. no. ab70562; Abcam), anti-yes-associated protein (YAP; 1:5,000; cat. no. ab205270; Abcam), anti-phosphorylated (p)-YAP (1:1,000; cat. no. ab205270; Abcam), anti-Bax (1:5,000; cat. no. ab32503; Abcam), anti-Bcl-2 (1:1,000; cat. no. ab182858; Abcam), anti-Ki-67 (1:1,000; cat. no. ab92742; Abcam) and anti-GAPDH (1:5,000; cat. no. ab9485; Abcam). Subsequently, the membranes were incubated with secondary antibodies (1:5,000; cat. nos. ab6721 and ab6789; Abcam) at room temperature for $1 \mathrm{~h}$. Bands were visualized by enhanced chemiluminescence (EMD Millipore). GAPDH was used as the loading control. Protein expression was quantified using Quantity One version 4.5 software (Bio-Rad Laboratories, Inc.).

Bioinformatic analysis. The gene expression profiles of 37 patients with OTSCC, including 20 pairs of tumor and adjacent nontumor tissues, were download from the Gene Expression Omnibus (GEO; GSE13601; https://www.ncbi.nlm. nih.gov/geo/) (15). This dataset contained the highest number of paired tissue expression data of OTSCC in GEO database. 

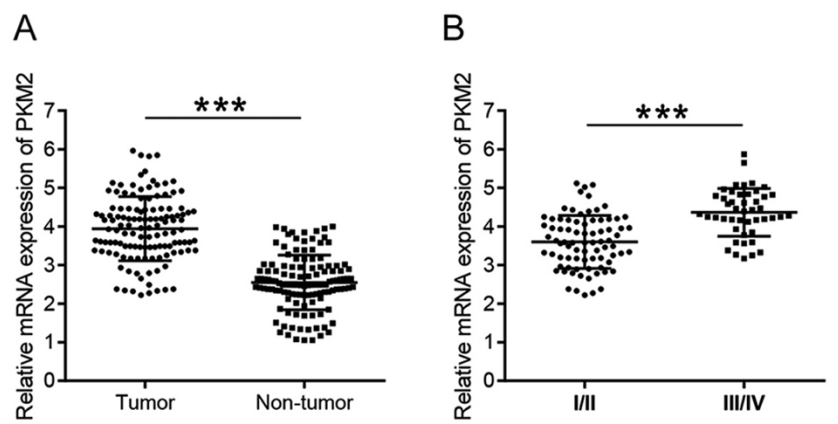
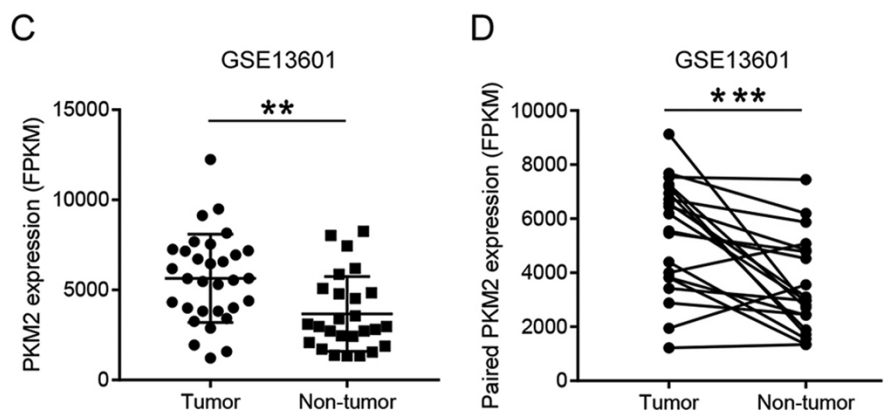

$\mathrm{E}$

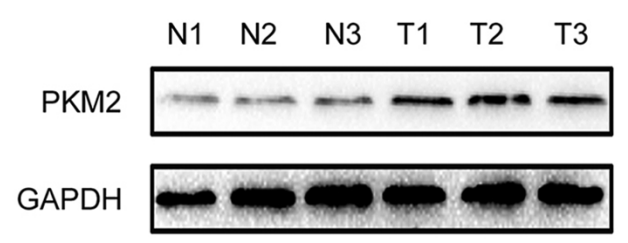

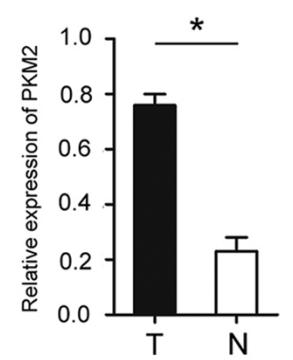

F

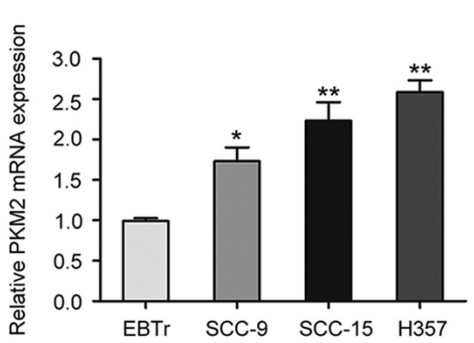

Figure 1. PKM2 expression in OTSCC tissues and cells. (A) Expression level of PKM2 in OTSCC and adjacent non-cancerous tissues. (B) Expression level of PKM2 in different stages of OTSCC. (C,D) PKM2 expression in OTSCC and paired adjacent non-cancerous tissues in the GSE13601 microarray dataset. (E) Protein expression of PKM2 in OTSCC and adjacent non-cancerous tissues. (F) Expression level of PKM2 in OTSCC cells. ${ }^{*} \mathrm{P}<0.05,{ }^{* *} \mathrm{P}<0.01$ and ${ }^{* * *} \mathrm{P}<0.001$. OTSCC, oral tongue squamous cell carcinoma; PKM2, pyruvate kinase M2; $\mathrm{N}$, normal; $\mathrm{T}$, tumor.

The data were normalized according to normalization function between Arrays function contained in limma package in R software (PMID: 25605792) (16), and the expression of PKM2 was selected and visualized by GraphPad Prism software. The clinicopathological parameters of patients included in this dataset are presented in Table SI.

CCK-8 assay. Cells were seeded in a 96-well plate at the density of $1 \times 10^{3}$ cells per $100 \mu \mathrm{l}$ and cultured for $24 \mathrm{~h}$ at $37^{\circ} \mathrm{C}$. Cells were incubated with $10 \mu \mathrm{l}$ of Cell Counting Kit-8 (CCK-8) solution (Dojindo Molecular Technologies, Inc.) for $1 \mathrm{~h}$. The absorbance was read at $450 \mathrm{~nm}$ using a microplate reader.

5-Ethynyl-2'-deoxyuridine (EdU) assay. Cells were seeded in a 96-well plate ( $1 \times 10^{5}$ cells) and cultured until they reached $30-50 \%$ confluence. Cells were fixed with $4 \%$ polyformaldehyde at room temperature for $30 \mathrm{~min}$ and the nuclei were permeabilized using $0.5 \%$ Triton X-100 solution at room temperature for $30 \mathrm{~min}$. Cells were incubated with EdU $(50 \mu \mathrm{M}), 1 \mathrm{x}$ ApolloR reaction cocktail $(100 \mu \mathrm{l})$ and $1 \mathrm{x}$ Hoechst $33342(100 \mu \mathrm{l})$ at room temperature for $30 \mathrm{~min}$ according to the manufacturer's instructions. Cells were visualized using fluorescent microscope (magnification, x100). Cell proliferation was analyzed according to the mean number of stained cells by using ImageJ (version 1.41; National Institutes of Health).

Flow cytometry analysis of cell apoptosis and cell cycle. To evaluate cell apoptosis, SCC-9 or H357 cells ( $1 \times 10^{5}$ cells) was seeded into 6 well plates. Once they reached confluence, cells were collected and incubated with Annexin V-FITC $(5 \mu 1)$ and propidium iodide (PI) solution (5 $\mu \mathrm{l}$; Biogot Technology Co., Ltd.) at room temperature for $15 \mathrm{~min}$ according to the manufacturers' instructions. Cells were subsequently suspended in
$400 \mu \mathrm{l}$ binding buffer. To evaluate the cell cycle, SCC-9 and $\mathrm{H} 357$ cells were collected and fixed in $75 \%$ ethanol at $-20^{\circ} \mathrm{C}$ overnight. Then, the fixed cells were washed with PBS and incubated with RNase A for $20 \mathrm{~min}$ at room temperature. These cells were stained with PI and incubated in the dark for $30 \mathrm{~min}$ at $4^{\circ} \mathrm{C}$. Cell apoptosis and cell cycle progression were analyzed using flow cytometry (BD Biosciences). The percentages of cells within each phase of the cell cycle were analyzed with ModFit version 4.0 (Verity Software House, Inc.) and CellQuest version 5.1 (Thermo Fisher Scientific, Inc.).

Statistical analysis. The data were analyzed using SPSS version 20.0 software (IBM Corp.) and GraphPad Prism version 6.0 software (GraphPad Software Inc.). The overall survival (OS) of patients with OTSCC was analyzed using Kaplan-Meier method and log-rank test. Univariate and multivariate cox regression analyses were used to analyze the prognostic significance of PKM2 expression. The association between PKM2 expression and the clinicopathological characteristics of patients with OTSCC patients was analyzed using a $\chi^{2}$ test. Differences between two groups were analyzed using Student's t-test. Comparisons between multiple groups were performed using one-way ANOVA followed by Tukey's post hoc test. $\mathrm{P}<0.05$ was considered to indicate a statistically significant difference.

\section{Results}

PKM2 expression in OTSCC. The expression of PKM2 in OTSCC and normal adjacent tissues was determined by RT-qPCR. The results demonstrated that PKM2 expression was significantly higher in OTSCC tissues compared with paired normal adjacent tissues (Fig. 1A). Furthermore, PKM2 
A

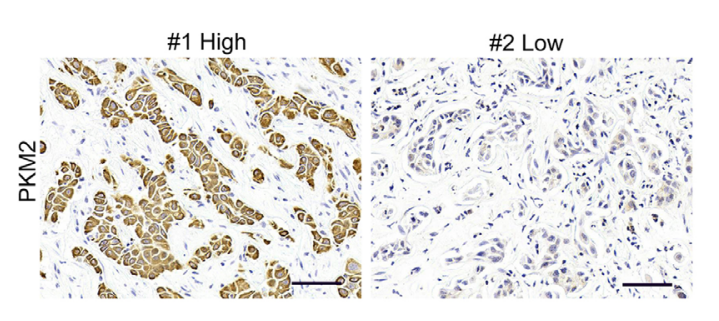

B

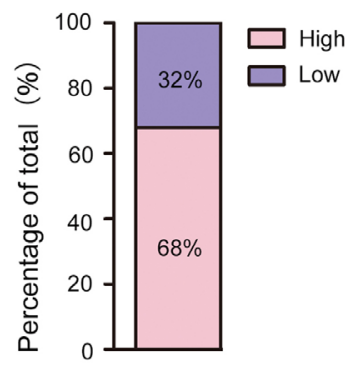

C

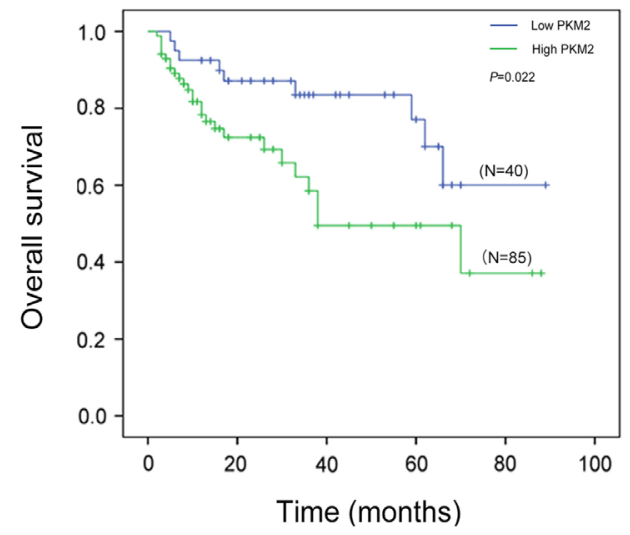

Figure 2. High expression of PKM2 predicts poor prognosis in patients with OTSCC. (A) Representative image of high and low expression of PKM2 in OTSCC tissues. (B) Statistical analysis of the results from immunohistochemical detection of PKM2 expression. (C) Kaplan-Meier analysis was used to determine the overall survival of PKM2 in patients with OTSCC. Scale bar=50 $\mu \mathrm{m}$. OTSCC, oral tongue squamous cell carcinoma; PKM2, pyruvate kinase M2.

Table I. Association between PKM2 expression and the clinicopathological characteristics of patients with oral tongue squamous cell carcinoma.

PKM2 expression

Variable n Low $(\mathrm{n}=40) \quad$ High $(\mathrm{n}=85) \quad$ P-value

Age, years

$\begin{array}{llll}<55 & 66 & 21 & 45 \\ \geq 55 & 59 & 19 & 40\end{array}$

Sex

$\begin{array}{llll}\text { Male } & 79 & 27 & 52 \\ \text { Female } & 46 & 13 & 33\end{array}$

Smoking

$\begin{array}{llll}\text { No } & 38 & 14 & 2 \\ \text { Yes } & 87 & 26 & 6\end{array}$

Drinking

$\begin{array}{llll}\text { No } & 45 & 15 & 30 \\ \text { Yes } & 80 & 25 & 55\end{array}$

TNM stage

$\begin{array}{llll}\mathrm{I} / \mathrm{II} & 61 & 28 & 32\end{array}$

III/IV $\quad 65 \quad 12 \quad 53$

Tumor size

$\begin{array}{llll}\text { T1-T2 } & 68 & 26 & 42 \\ \text { T3-T4 } & 57 & 14 & 43\end{array}$

PKM2, oral tongue squamous cell carcinoma; TNM, Tumor-NodeMetastasis. expression was significantly higher in stage III/IV OTSCC samples compared with I/II OTSCC samples (Fig. 1B). Similar results were obtained by analyzing microarray sequencing results (GSE13601) downloaded from GEO public database using Graphpad Prism software (Fig. 1C and D). The results from western blotting confirmed that PKM2 was highly expressed in OTSCC tissues compared with normal tissues (Fig. 1E). The expression of PKM2 was also significantly increased in OTSCC cell lines compared with normal cells (Fig. 1F).

Association between PKM2 expression and the clinicopathological characteristics of patients with OTSCC. The results from IHC staining demonstrated that PKM2 expression was high in 85 out of the 125 OTSCC samples (68\%) and low in 40 out of 125 OTSCC samples (32\%; Fig. 2A and B). Furthermore, high PKM2 expression was associated with 0.881 TNM stage (Table I). However, there was no significant association between PKM2 expression and age, sex, smoking, drinking or tumor size (all $\mathrm{P}>0.05$ ).

Association between PKM2 expression and OS in patients with OTSCC. The association between PKM2 expression and the prognosis of patients with OTSCC was determined. The results from Kaplan-Meier survival analysis revealed that patients with high PKM2 expression had significantly shorter OS compared with those with low PKM2 expression (Fig. 2C). In addition, univariate analysis demonstrated that TNM stage $[\mathrm{P}=0.009$; confidence interval $(\mathrm{CI}): 1.045-4.669$, hazard ratio $(\mathrm{HR})=1.473]$ and $\mathrm{PKM} 2$ expression $(\mathrm{P}=0.007$; CI:1.047-3.647; 
Table II. Univariate and multivariate analysis of the clinicopathological characteristics of patients with oral tongue squamous cell carcinoma.

\begin{tabular}{|c|c|c|c|c|c|}
\hline \multirow[b]{2}{*}{ Variable } & \multirow[b]{2}{*}{$\mathrm{n}$} & \multicolumn{2}{|c|}{ Univariate analysis } & \multicolumn{2}{|c|}{ Multivariate analysis model } \\
\hline & & $\mathrm{HR}(95 \% \mathrm{CI})$ & P-value & $\mathrm{HR}(95 \% \mathrm{CI})$ & P-value \\
\hline Sex & & $0.364(0.408-1.273)$ & 0.534 & & \\
\hline Male & 70 & & & & \\
\hline Female & 46 & & & & \\
\hline Age, years & & $0.475(0.339-1.567)$ & 0.663 & & \\
\hline$<55$ & 66 & & & & \\
\hline$\geq 55$ & 59 & & & & \\
\hline Smoking & & $1.203(0.963-3.442)$ & 0.076 & & \\
\hline No & 38 & & & & \\
\hline Yes & 87 & & & & \\
\hline Drinking & & $1.104(0.861-2.776)$ & 0.537 & & \\
\hline No & 45 & & & & \\
\hline Yes & 80 & & & & \\
\hline TNM stage & & $1.473(1.045-4.669)$ & 0.009 & $1.186(0.978-2.604)$ & 0.011 \\
\hline I/II & 61 & & & & \\
\hline III/IV & 65 & & & & \\
\hline Tumor size & & $0.864(0.883-1.487)$ & 0.086 & & \\
\hline $\mathrm{T} 1-\mathrm{T} 2$ & 68 & & & & \\
\hline T3-T4 & 57 & & & & \\
\hline PKM2 expression & & $1.227(1.047-3.647)$ & 0.007 & $1.377(0.768-2.964)$ & 0.013 \\
\hline High & 85 & & & & \\
\hline Low & 40 & & & & \\
\hline
\end{tabular}

PKM2, oral tongue squamous cell carcinoma; TNM, Tumor-Node-Metastasis; CI, confidence interval.

$\mathrm{HR}=1.227$ ) were significantly associated with OS in patients with OTSCC (Table II). The results from multivariate analysis demonstrated that TNM stage $(\mathrm{P}=0.011$; CI, 0.978-2.604; $\mathrm{HR}=1.186)$ and $\mathrm{PKM} 2$ expression $(\mathrm{P}=0.013$; $\mathrm{CI}, 0.768-2.964$; $\mathrm{HR}=1.377$ ) were independent prognostic factors for OS in patients with OTSCC (Table II).

PKM2 knockdown inhibits OTSCC cell proliferation. To determine the effect of PKM2 on OTSCC cell proliferation, CCK-8 assay and EdU uptake experiments were performed. Transfection with siRNA-PKM2 was used to significantly decrease PKM2 expression in SCC-9 and H357 cells (Fig. 3A and B). The results from CCK-8 assay revealed that PKM2 downregulation significantly inhibited SCC-9 and $\mathrm{H} 357$ cell proliferation (Fig. 3C and D). Furthermore, as presented in Fig. 3E and F, the number of SCC-9 and H357 cells incorporating EdU was significantly lower in PKM2 knockdown group compared with the control group.

PKM2 knockdown inhibits cell cycle and induces apoptosis of OTSCC cells. The effects of PKM2 on cell cycle and apoptosis of OTSCC cells were analyzed. The results from flow cytometry demonstrated that PKM2 silencing induced a significant increase in SCC-9 and $\mathrm{H} 357$ cell apoptotic rate (Fig. 4A and B). In addition, the number of cells in G1 phase was increased, while the number of cells in $\mathrm{S}$ phase was decreased in si-PKM2 transfected cells compared with control cells (Fig. 4C and D).

PKM2 knockdown modulates the activation of the Hippo pathway in OTSCC cells. To determine the specific mechanism by which PKM2 might affect the proliferation and apoptosis of OTSCC cells, the expression of proteins from the Hippo signaling pathway and of proteins associated with cell proliferation and apoptosis was determined. The results demonstrated that PKM2 silencing significantly decreased the protein expression of YAP, Bcl-2 and Ki-67, and significantly enhanced the protein expression of LATS1, p-YAP and Bax in SCC-9 and H357 cells (Fig. 5A and B). Taken together, these findings suggested that PKM2 may exert its function on OTSCC cell proliferation and apoptosis in part through the Hippo pathway.

\section{Discussion}

Oral cancer is the general term for malignant tumors that occur in the oral cavity. Each year, 300,000 new cases are diagnosed and this cancer ranks sixth among all malignant tumors (17). OTSCC is a common form of oral cancer that occurs in the gums, lips, tongue, soft and hard palate or throat, with strong migration and invasion ability. OTSCC seriously endanger human health (18). At present, a comprehensive 
A

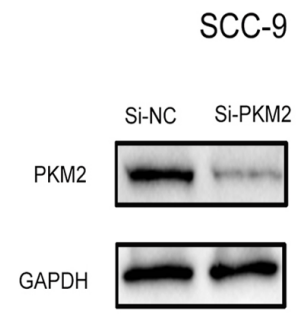

C

SCC-9

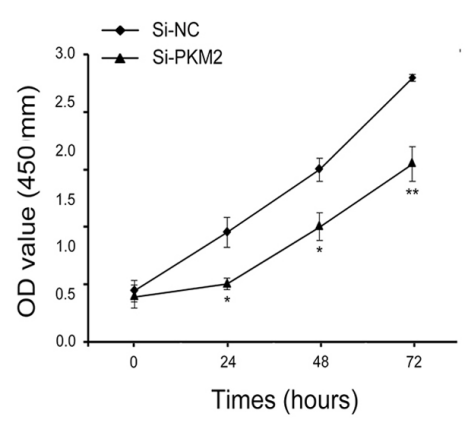

$\mathrm{E}$

SCC-9

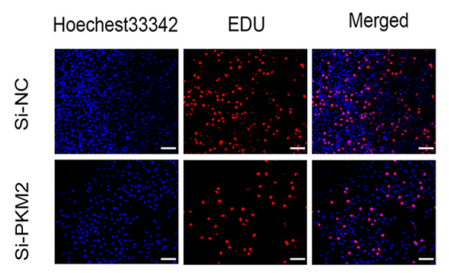

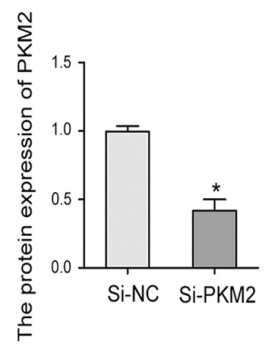

B

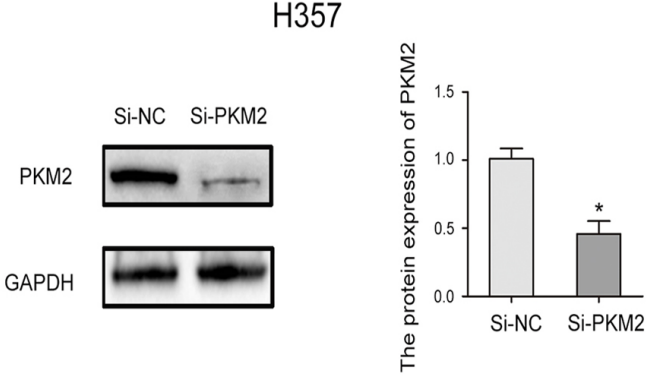

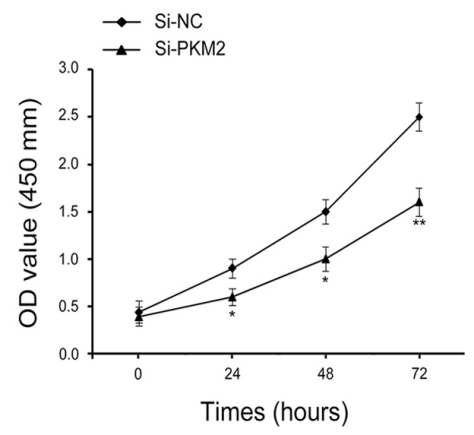

$\mathrm{F}$

\section{$\mathrm{H} 357$}

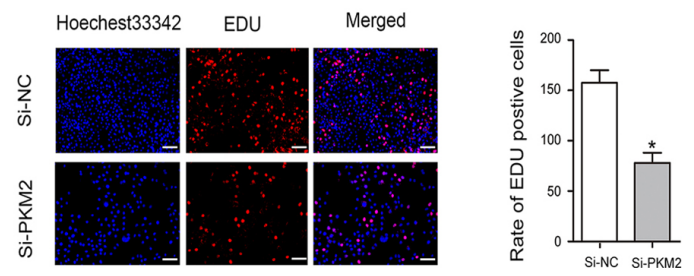

Figure 3. Effect of PKM2 on the proliferation of OTSCC cells. (A) Protein expression of PKM2 in SCC-9 cells following PKM2 silencing. (B) Protein expression of PKM2 in H357 cells following PKM2 silencing. (C) CCK-8 assay was performed to examine the effects of PKM2 on SCC-9 cell proliferation. (D) CCK-8 assay was performed to examine the effects of PKM2 on H357 cell proliferation. (E) Cell proliferation was detected with EdU in SCC-9 following PKM2 silencing. (F) Cell proliferation was detected with EdU in H357 cells following PKM2 silencing. Scale bar, $100 \mu \mathrm{m}$. ${ }^{*} \mathrm{P}<0.05$ and ${ }^{* *} \mathrm{P}<0.01$. OTSCC, oral tongue squamous cell carcinoma; PKM2, pyruvate kinase M2; si, small interfering; NC, negative control; OD, optical density; CCK-8, Cell Counting Kit-8.

treatment approach is typically adopted for patients with OTSCC and includes a combination of surgery, radiotherapy and chemotherapy. Although surgery can remove tumors visible to the naked eye and can be combined with radiotherapy and chemotherapy, active tongue movement, abundant blood circulation and lymphatic reflux often lead to poor prognosis and short-term survival (19). Due to the rapid growth of OTSCC, the strong infiltration and the easy development of resistance to radiotherapy and chemotherapy, the 5-year survival rate is only 50\% (20). Recently, studies have reported that the occurrence and development of oral squamous cell carcinoma is a complex process involving multiple genes, multiple steps and multiple stages $(21,22)$. Given the increasing interest in molecular targeted therapies for oral cancer, it is urgent to identify novel molecular targets that would allow the accurate diagnosis and treatment of OTSCC, in order to improve the survival time and quality of life of patients with OTSCC.

The Warburg effect is the main way for generating ATP in tumor cells via mitochondrial oxidative phosphorylation of glucose produced by aerobic glycolysis. The Warburg effect is an essential component of the metabolic rearrangement of tumor cells (23). An important feature of tumor cells is that under normal oxygen content, glucose intake and lactic acid accumulation will also gradually increase (24). As glycolysis is the main source of energy metabolism, a higher 


\section{A}

SCC-9
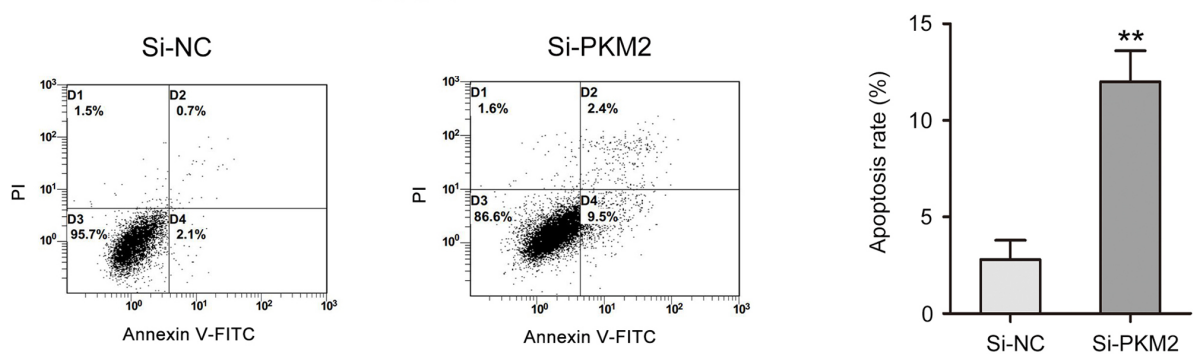

B

$\mathrm{H} 357$
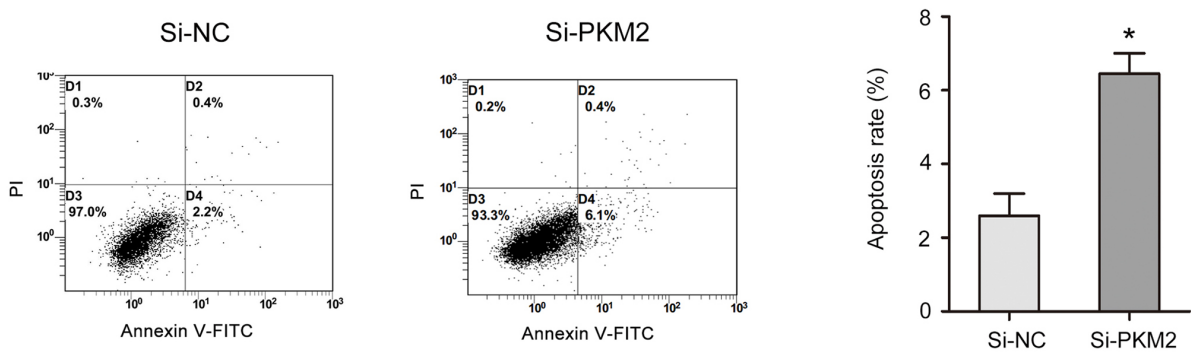

C

SCC-9
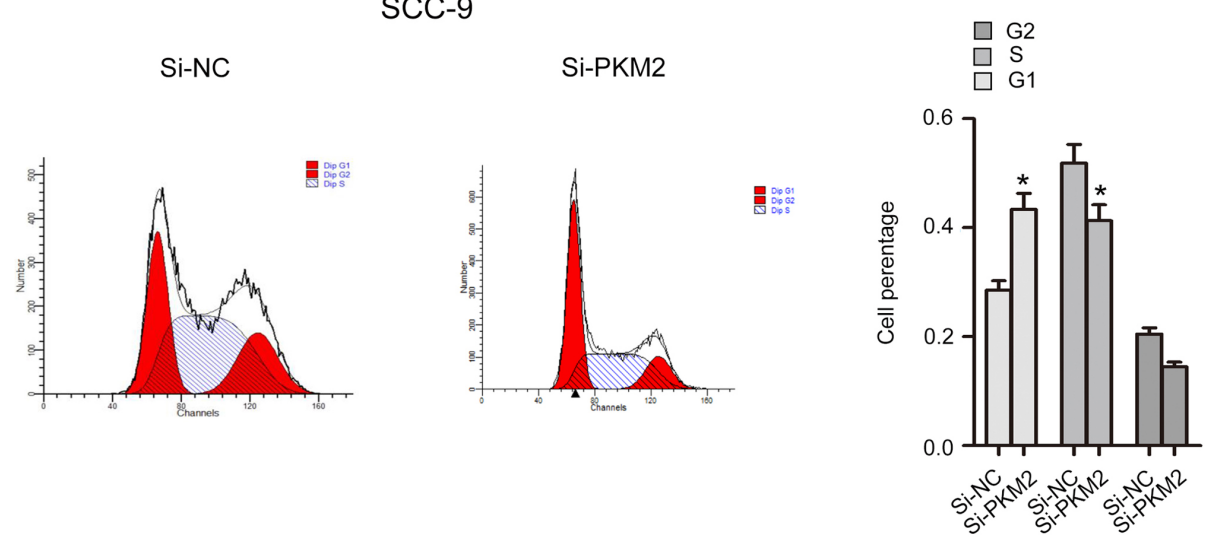

D

H357

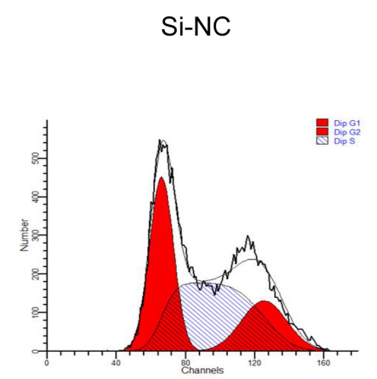

Si-PKM2

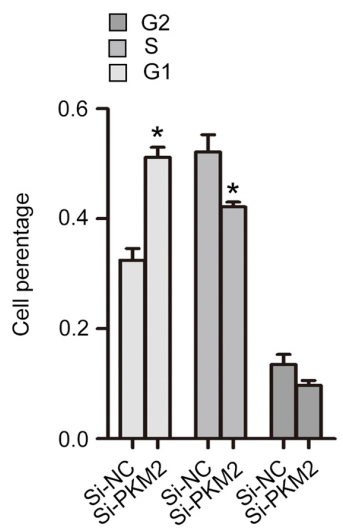

Figure 4. Effect of PKM2 on the cell cycle and apoptosis of OTSCC cells. (A) Flow cytometry was used to examine the effects of PKM2 silencing on SCC-9 cell apoptosis. (B) Flow cytometry was used to examine the effects of PKM2 silencing on H357 cell apoptosis. (C) Flow cytometry was used to compare the effects of PKM2 downregulation on the cell cycle distribution in SCC-9 cells. (D) Flow cytometry was used to compare the effects of PKM2 downregulation on the cell cycle distribution in $\mathrm{H} 357$ cells. ${ }^{*} \mathrm{P}<0.05$ and ${ }^{* *} \mathrm{P}<0.01$. OTSCC, oral tongue squamous cell carcinoma; PKM2, pyruvate kinase M2; si, small interfering; $\mathrm{NC}$, negative control. 
A

\section{SCC-9}

Si-NC Si-PKM2

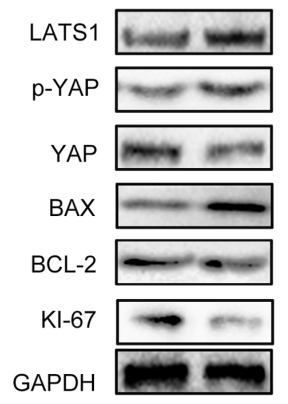

B
Si-PKM2 $\square$

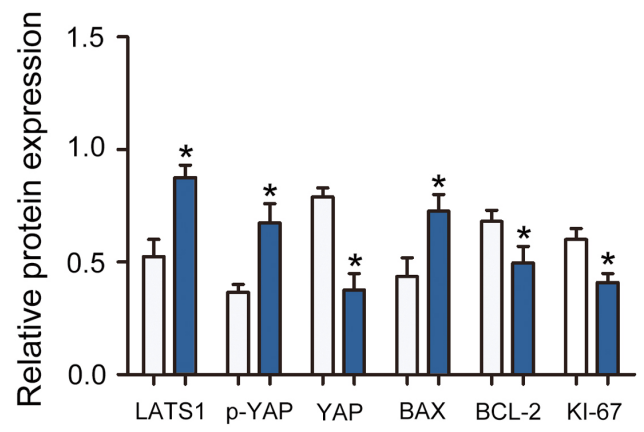

\section{H357}

Si-PKM2 $\square$
Si-NC $\square$
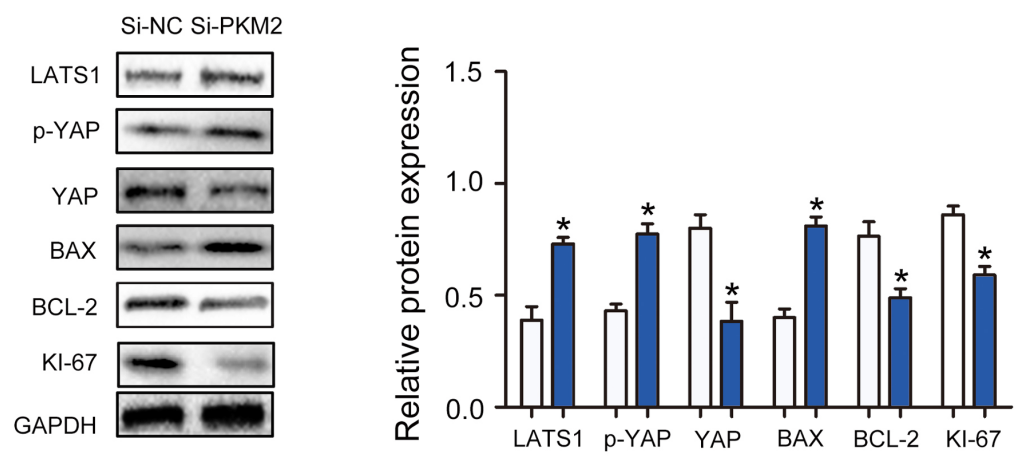

Figure 5. Effect of PKM2 on the expression of proteins from the Hippo signaling pathway and of proteins associated with cell proliferation and apoptosis. (A) Protein expression of Hippo signaling pathway components, including proliferation- and apoptosis-related proteins in SCC-9 cells following PKM2 silencing. (B) Protein expression of Hippo signaling pathway components, including proliferation- and apoptosis-related proteins in $\mathrm{H} 357$ cells following PKM2 silencing. "P<0.05. PKM2, pyruvate kinase M2; si, small interfering; NC, negative control.

glycolytic capacity is obtained and glucose is converted into lactic acid to produce ATP (25). The Warburg effect therefore allows tumor cells to replenish their productivity under the microenvironment, which is characterized by local hypoxia and inhibition of mitochondrial oxidative phosphorylation. The Warburg effect generates a high ATP/ADP ratio to allow the rapid proliferation of tumor cells via macromolecular anabolism to promote malignant signal transduction mechanisms maintained by tumor cells (26). Previous studies have confirmed that effective inhibition of the Warburg effect can significantly inhibit the malignant growth of tumors and increase the level of apoptosis of tumor cells $(27,28)$. The Warburg effect is essential for the energy metabolism rearrangement of tumor cells, although its underlying mechanism requires further investigation.
PK is the key enzyme of glycolysis and it catalyzes the formation of pyruvate from phosphoenolpyruvate $(29,30)$. There are four major PK isoenzymes, named PKL, PKR, PKM1 and PKM2, which are tissue specific (31). The expression level of PKM2 has been associated with the clinical stage and pathological grades of various types of tumor. PKM2 activates $>100$ proteins, including ERK1/2 (32). Sustained activation of PKM2 promotes the proliferation and migration of tumor cells. Mitomycin 2, which is a key regulator of mitochondrial fusion, interacts with PKM2, which promotes mitochondrial fusion and production of phosphorus oxide that weakens glycolysis (33). In ovarian cancer, PKM2 overexpression leads to increased cell proliferation and tumor growth, which might be related to the effect of PKM2 on cell cycle progression, such as the decrease in G1 phase and the significant increase in S phase (34). PKM2 
is transported from the cytoplasm to the mitochondria in case of increased oxidative stress (35). Once in the mitochondria, PKM2 interacts with Bcl-2 and phosphorylates Bcl-2 at T69 site. This phosphorylation blocks the binding of Cul3-based E3 ligase to $\mathrm{Bcl}-2$ and subsequent degradation of $\mathrm{Bcl}-2$, and significantly inhibits the malignant proliferation of tumor cells (35). Similarly, glycogen synthetase kinase-3 $\beta$ forms protein complexes with Hsp90 and PKM2, which directly mediate the phosphorylation of T328 by Hsp90. T328 phosphorylation is essential to maintain the stability of PKM2, promote the glycolysis and proliferation of HCC cells, and inhibit apoptosis (36). miR-138 regulates the expression of PKM2 to mediate superoxide dismutase 2 activity and intracellular $\mathrm{H}_{2} \mathrm{O}_{2}$ levels to enhance the metastatic potential of tongue squamous cell carcinoma (37). In head and neck squamous cell carcinoma, the abnormal expression of PKM2 is associated with a poor prognosis. In particular, nuclear PKM2 activates $\beta$-catenin signaling and influences the proliferation and chemotherapeutic sensitivity of head and neck squamous cell carcinoma cells (38). In the biological process of tongue squamous cell carcinoma growth, invasion and apoptosis, PKM2 expression is increased, reflecting the higher energy flow of tumor cells and the dependence of their metabolism on aerobic glycolysis and oxidative phosphorylation. The expression of PKM2 is also related to the production of reactive oxygen species, glutamine and lactic acid. It has been reported that PKM2 has multiple tumor progression functions in oral squamous cell carcinoma (39). Taken together, these observations suggest that PKM2 might be considered as an oncogene involved in the promotion of tumor occurrence and development.

In the GEO database, the highest number of paired OTSCC and normal adjacent tissues was seen in the GSE13601 dataset, whereas only 4-12 paired mRNA expression profile was included in other datasets (GSE3524, GSE2280 and GSE138206, GSE9844). In addition, some OTSCC datasets only contained miRNA or long non-coding-RNA expression profiles, such as GSE51829, GSE51700, GSE98463, GSE137865, and some datasets only contained tumor mRNA expression file. Subsequently, GSE13601 was the most appropriate dataset to be used in the present study. The association between PKM2 expression and the clinicopathological characteristics of patients with OTSCC included in this dataset was therefore analyzed. The results demonstrated that PKM2 was highly expressed in patients with OTSCC. In addition, patients with OTSCC and high PKM2 expression had worse OS, and high PKM2 expression was associated with TNM stage. TNM stage and PKM2 expression were therefore considered as independent prognostic factors for OS in OTSCC. In addition, the results from in vitro experiments demonstrated that PKM2 knockdown significantly inhibited the proliferation and increased the apoptosis of OTSCC cells.

In summary, the findings from the present study suggested that the high expression of PKM2 in OTSCC tissues may be considered as an independent risk factor for the prognosis of patients with OTSCC, and that PKM2 may therefore serve a crucial role in promoting the proliferation of OTSCC cells.

\section{Acknowledgements}

Not applicable.

\section{Funding}

This work was supported by the Key Research and Development Projects of Sichuan Science and Technology Department (grant no. 2018FZ0113), the China Stomatological Association Western Medicine Stomatology Clinical Research Fund Project (grant no. CSA-W2016-01), the Sichuan Science and Technology Support Program (grant no. 2014SZ0038) and the Sichuan Provincial Primary Health Service Development Research Fund (grant no. SWFZ20-C-086).

\section{Availability of data and materials}

All data generated or analyzed during the present study are included in this published article.

\section{Authors' contributions}

JL and SY conducted the experiments, analyzed the data and wrote the manuscript. JL, LG and LZ conceived the study and revised the manuscript critically for important intellectual content. LG and SY made substantial contributions to patient and tissue specimen collection and data interpretation. JL and SY confirm the authenticity of all the raw data. All authors read and approved the final manuscript.

\section{Ethics approval and consent to participate}

The present study was approved by the Research Ethics Committee of Suining Central Hospital (approval no. 20110067). The patients signed written informed consent. All specimens were handled and anonymized according to ethical and legal standards.

\section{Patient consent for publication}

Not applicable.

\section{Competing interests}

The authors declare that they have no competing interests.

\section{References}

1. Siegel RL, Miller KD and Jemal A: Cancer statistics, 2015. Cancer J Clin 65: 5-29, 2015.

2. Chi AC, Day TA and Neville BW: Oral cavity and oropharyngeal squamous cell carcinoma-an update. Cancer J Clin 65: 401-421, 2015.

3. Gillison ML, Chaturvedi AK, Anderson WF and Fakhry C: Epidemiology of human papillomavirus-positive head and neck squamous cell carcinoma. J Clin Oncol 33: 32-37, 2015.

4. Siegel RL, Miller KD and Jemal A: Cancer statistics, 2018. Cancer J Clin 68: 7-30, 2018.

5. Tamada M, Suematsu M and Saya H: Pyruvate kinase M2: Multiple faces for conferring benefits on cancer cells. Clin Cancer Res 18: 5554-5561, 2012.

6. Luo W and Semenza GL: Emerging roles of PKM2 in cell metabolism and cancer progression. Trends Endocrinol Metab 23: 560-566, 2012.

7. Dayton TL, Jacks T and Vander Heiden MG: PKM2, cancer metabolism, and the road ahead. EMBO Rep 17: 1721-1730, 2016

8. Christofk HR, Vander Heiden MG, Harris MH, Ramanathan A, Gerszten RE, Wei R, Fleming MD, Schreiber SL and Cantley LC: The M2 splice isoform of pyruvate kinase is important for cancer metabolism and tumour growth. Nature 452: 230-233, 2008. 
9. Burns JS and Manda G: Metabolic pathways of the Warburg effect in health and disease: Perspectives of choice, chain or chance. Int J Mol Sci 18: 2755, 2017.

10. Zhou ZF, Li M, Zhang L, Zhao H, Şahin Ö, Chen J, Zhao JJ, Songyang $\mathrm{Z}$ and $\mathrm{Yu} \mathrm{D}$ : Oncogenic kinase-induced PKM2 tyrosine 105 phosphorylation converts nononcogenic PKM2 to a tumor promoter and induces cancer stem-like cells. Cancer Res 78: 2248-2261, 2018

11. Liu FB, Ma FF, Wang YY, Hao L, Zeng H, Jia C, Wang Y, Liu P, Ong IM, Li B, et al: PKM2 methylation by CARM1 activates aerobic glycolysis to promote tumorigenesis. Nat Cell Biol 19: 1358-1370, 2017.

12. Li M, Bai YT, Han K, Li XD and Meng J: Knockdown of ectodysplasin-A receptor-associated adaptor protein exerts a tumor-suppressive effect in tongue squamous cell carcinoma cells. Exp Ther Med 19: 3337-3347, 2020.

13. Livak KJ and Schmittgen TD: Analysis of relative gene expression data using real-time quantitative PCR and the 2(-Delta Delta C(T)) method. Methods 25: 402-408, 2001

14. Wu H, Zhang W, Wu Z, Liu Y, Shi Y, Gong J, Shen W and Liu C: miR-29c-3p regulates DNMT3B and LATS1 methylation to inhibit tumor progression in hepatocellular carcinoma. Cell Death Dis 10: 48, 2019.

15. Estilo CL, O-charoenrat P, Talbot S, Socci ND, Carlson DL, Ghossein R, Williams T, Yonekawa Y, Ramanathan Y, Boyle JO, et al: Oral tongue cancer gene expression profiling: Identification of novel potential prognosticators by oligonucleotide microarray analysis. BMC Cancer 9: 11, 2009.

16. Ritchie ME, Phipson B, Wu D, Hu Y, Law CW, Shi W and Smyth GK: Limma powers differential expression analyses for RNA-sequencing and microarray studies. Nucleic Acids Res 43: e47, 2015.

17. Siegel RL, Miller KD and Jemal A: Cancer statistics, 2019. Cancer J Clin 69: 7-34, 2019.

18. Solomon B, Young RJ and Rischin D: Head and neck squamous cell carcinoma: Genomics and emerging biomarkers for immunomodulatory cancer treatments. Semin Cancer Biol 52: 228-240, 2018

19. Omura K: Current status of oral cancer treatment strategies: Surgical treatments for oral squamous cell carcinoma. Int J Clin Oncol 19: 423-430, 2014

20. Ali J, Sabiha B, Jan HU, Haider SA, Khan AA and Ali SS: Genetic etiology of oral cancer. Oral Oncol 70: 23-28, 2017.

21. Hussein AA, Forouzanfar T, Bloemena E, de Visscher J, Brakenhoff RH, Leemans CR and Helder MN: A review of the most promising biomarkers for early diagnosis and prognosis prediction of tongue squamous cell carcinoma. Br J Cancer 119: 724-736, 2018.

22. Kim YJ and Kim JH: Increasing incidence and improving survival of oral tongue squamous cell carcinoma. Sci Rep 10: 7877,2020

23. Lu J: The Warburg metabolism fuels tumor metastasis. Cancer Metastasis Rev 38: 157-164, 2019.

24. Icard P, Shulman S, Farhat D, Steyaert JM, Alifano M and Lincet H: How the Warburg effect supports aggressiveness and drug resistance of cancer cells? Drug Resist Updat 38: 1-11, 2018.
25. Wilde L, Roche M, Domingo-Vidal M, Tanson K, Philp N, Curry J and Martinez-Outschoorn U: Metabolic coupling and the reverse Warburg effect in cancer: Implications for novel biomarker and anticancer agent development. Semin Oncol 44: 198-203, 2017.

26. Liberti MV and Locasale JW: The Warburg effect: How does it benefit cancer cells? Trends Biochem Sci 41: 211-218, 2016.

27. Vander Heiden MG, Cantley LC and Thompson CB: Understanding the Warburg Effect: The metabolic requirements of cell proliferation. Science 324: 1029-1033, 2009.

28. Sun L, Suo C, Li ST, Zhang H and Gao P: Metabolic reprogramming for cancer cells and their microenvironment: Beyond the Warburg Effect. Biochim Biophys Acta Rev Cancer 1870: 51-66, 2018.

29. Wiese EK and Hitosugi T: Tyrosine kinase signaling in cancer metabolism: PKM2 paradox in the Warburg effect. Front Cell Dev Bio 6: 79, 2018.

30. van Niekerk G and Engelbrecht AM: Role of PKM2 in directing the metabolic fate of glucose in cancer: A potential therapeutic target. Cell Oncol (Dordr) 41: 343-351, 2018.

31. Zhang Z, Deng X, Liu Y, Liu Y, Sun L and Chen F: PKM2, function and expression and regulation. Cell Biosci 9: 52, 2019.

32. Yang W, Zheng Y and Xia Y: ERK1/2-dependent phosphorylation and nuclear translocation of PKM2 promotes the Warburg effect. Nat Cell Biol 14: 1295-1304, 2012.

33. Li T, Han J, Jia L, Hu X, Chen L and Wang Y: PKM2 coordinates glycolysis with mitochondrial fusion and oxidative phosphorylation. Protein Cell 10: 583-594, 2019.

34. Miao Y, Lu M, Yan Q, Li SD and Feng YJ: Inhibition of proliferation, migration, and invasion by knockdown of pyruvate Kinase-M2 (PKM2) in ovarian cancer SKOV3 and OVCAR3 Cells. Oncol Res 24: 463-475, 2016.

35. Liang J, Cao R, Wang X, Zhang Y, Wang P, Gao H, Li C, Yang F, Zeng $\mathrm{R}$, Wei P, et al: Mitochondrial PKM2 regulates oxidative stress-induced apoptosis by stabilizing Bcl2. Cell Res 27: 329-351, 2017.

36. Xu Q, Tu J, Dou C, Zhang J, Yang L, Liu X, Lei K, Liu Z, Wang Y, $\mathrm{Li}$ L, et al: HSP90 promotes cell glycolysis, proliferation and inhibits apoptosis by regulating PKM2 abundance via Thr-328 phosphorylation in hepatocellular carcinoma. Mol Cancer 16: 178, 2017.

37. Wang W, He Q, Sun J, Liu Z, Zhao L, Lu Z, Zhou X and Wang A: Pyruvate kinase M2 deregulation enhances the metastatic potential of tongue squamous cell carcinoma. Oncotarget 8: 68252-68262, 2017.

38. Jing C, Qu X, Li Z, Wu C, Zhao M, Wang Y, Sun S, Zhang S, Chen J, Qiao Y, et al: EGFRwt/vIII-PKM2- $\beta$-catenin cascade affects proliferation and chemo-sensitivity in head and neck squamous cell carcinoma. Am J Cancer Res 7: 2491-2502, 2017.

39. Kurihara-Shimomura M, Sasahira T, Nakashima C, Kuniyasu H Shimomura $\mathrm{H}$ and Kirita T: The Multifarious functions of pyruvate kinase M2 in oral cancer cells. Int J Mol Sci 19: 2907, 2018.

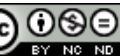

This work is licensed under a Creative Commons Attribution-NonCommercial-NoDerivatives 4.0 International (CC BY-NC-ND 4.0) License. 